\title{
Comprehensive analysis of Pisum sativum (L.) microRNAs
}

\author{
Kulaeva O.A. ${ }^{1 *}$, Zorin E.A. ${ }^{1}$, Romanyuk D.A. ${ }^{1}$, Gribchenko E.S. ${ }^{1}$, Afonin A.M. ${ }^{1}$, \\ Gordon M.L. ${ }^{1}$, Shtark O.Y. ${ }^{1}$, Tikhonovich I.A. ${ }^{1,2}$, Zhukov V.A. ${ }^{1}$ \\ ${ }^{1}$ All-Russia Research Institute for Agricultural Microbiology, St. Petersburg, Russia \\ ${ }^{2}$ Saint-Petersburg State University, St. Petersburg, Russia \\ *email: okulaeva@arriam.ru
}

Various processes of plant development, biotic and abiotic interactions occur with participation of different regulators, one of the main ones are microRNAs. To date, there is very small information in literature about microRNAs of garden pea (Pisum sativum L.) which among the valuable pulse crops all over the world. To increase information about pea microRNAs, Next Generation Sequencing (NGS) technologies were used. As a result, sets of microRNAs from lower and upper parts of plants were identified and annotated. Analysis of degradome sequencing, which made it possible to identify cleavage sites in transcripts, revealed targets of these microRNAs. For some transcripts, regulation by more than one miRNA has been shown and some miRNAs are involved in the regulation of several transcripts. Then searching for microRNAs which can be used like reference genes was done. The analysis was carried out on plants grown under conditions of legume-rhizobia and mycorrhizal symbiosis. The effect of cadmium was also investigated. Analysis of set of microRNAs made by Real Time PCR (RT PCR) allowed to identify several microRNAs with stable expression level, as for all conditions and for different interaction processes separately. These microRNAs can be considered as reference sequences for the further study of expression by RT PCR. At the final stage of the work expression analysis of pea microRNAs was done by NGS and RT PCR. Certain pea microRNAs were shown to be involved in the processes of legume-rhizobia symbiosis, arbuscular mycorrhiza development and processes of plant adaptation to cadmium.

Acknowledgements: This work was supported by RFBR grant No. 20-04-01136 and by RFBR grant No. 19-316-90058. 